\title{
The Visual Form of Interaction from the Perspective of Aesthetics
}

\author{
Mohammad Bahramzadeh ${ }^{1}$, Maryam Ghorbani ${ }^{2}$, Sadaf Sabokrou ${ }^{3, *}$ \\ ${ }^{1}$ Archaeology Department, Islamic Azad University of Abhar, Abhar, Iran \\ ${ }^{2}$ Architecture Department, Payamnour University, Rasht, Iran \\ ${ }^{3}$ Architecture Department, Kadous Non-profit University, Rasht, Iran
}

\section{Email address:}

Mohammadbahramzadeh@gmail.com (M. Bahramzadeh), Maryam.ghorbanii@gmail.com (M. Ghorbani), sadaf_ar1382@yahoo.com (S. Sabokrou)

\section{To cite this article:}

Mohammad Bahramzadeh, Maryam Ghorbani, Sadaf Sabokrou. The Visual Form of Interaction from the Perspective of Aesthetics. International Journal of Science, Technology and Society. Special Issue: Research and Practice in Architecture and Urban Studies in Developing Countries. Vol. 3, No. 2-1, 2015, pp. 116-121. doi: 10.11648/j.ijsts.s.2015030201.33

\begin{abstract}
Addressing the issue of beautification of our city ensures comfort and security of citizens which can increase the efficiency of communal spaces. Aesthetics can be classified to sensory, form and symbolic aesthetics. Form is the sensible, characteristic and understandable identity of a phenomenon and is the effective factor in creating space. This study is an analytical and descriptive approach using existing resources in library studies and tries to study the visual forms of interaction from the aesthetics perspective by enumerating some of the principles and concepts and case studies.
\end{abstract}

Keywords: Aesthetics, Space, Form

\section{Introduction}

One of the ways to give identity to the cities and their sustainability is their skeleton-physical texture. Paying no attention to the coordinating criteria and principles in designing urban environments has led the physical space of the city to be appeared improper. Understanding the aesthetic aspects of cities and strengthening the aesthetic aspects of complexes might be a good start for addressing this issue. Paying attention to beautification of cities ensures the psychological comfort and security of its citizens, which increases the efficiency of the public space [1].

To express any spiritual content, proportionate and perceptible visual form must be created and so a need is felt for the language of forms. We achieve the sense of space seeing (reading) the visual forms. In this sense, expressing the concepts using the visual forms is easier than the written and spoken language.

Only in the mirror of urban landscape, the intangible aspects of civic life such as poverty and health, historical depth of city, aesthetic records of subcultures and... are manifested through a system of visual symbols and forms and provides the assessment of sustainability. Cities owning desirable urban design are able to improve the mental image if community from themselves and civic pride, broadening the aesthetic experience of citizens and by raising up the reputation and image of the city in the national and international level, they strengthen the competitive power of the city to attract more capital and creative sectors and consequently, result in the emergence of a sustainable city. [2]

Designing the city according to the aesthetic roots and principles in the world, is yet unknown from the general idea or the execution method point of view. In the United States, there were a number of examples from the 80 's and 90 's that there, some rules were presented in order to beautify. By the help of these criteria, the urban design became oriented and substitute of spontaneous urbanization. Kevin Lynch in 1960 put forward the suggestion of "visual plans". He believes that recommendations and controls can be suggested and applied about the visual and physical forms in the urban scale. [1]

In this paper, an analytical and descriptive approach using existing resources in library studies and tries to study the visual forms of interaction from the aesthetics perspective by enumerating some of the principles and concepts and case studies. 


\section{Definition of Aesthetics}

The word aesthetics is Greek in original and means perception. Science aesthetic broadly means to search for ways to feel the ambience and the position within it. [3] Moein Encyclopedia defines beauty as "beautiful manner and quality" and writes: It is the order and harmony accompanying the greatness and purity in the object, and stimulates the great intellect and imagination and brings the pleasure and relaxation. It is something relative. [4] Full understanding of aesthetics is difficult, but understanding the effective factors in understanding pleasant of the environment is possible.

George Santayana classifies the aesthetics into three sensory, form and symbolic aesthetics. Sensory aesthetics analysis are strongly internal and subjective. Symbolic aesthetic is deal with the pleasure from people's mental history and mentality of the configuration and characteristics of the built environment. The issue of form aesthetics is the role and effect of shapes, proportion, rhythm, scale, complexity, color and other elements of the built environment. [5]

What is the aim of aesthetics? Sigmund Freud knows the goal of beauty as enjoyment of art and somehow the emotional relief. According to Theodor Adorno, aesthetic behavior is the ability to understand the object more than what the objects are. According to information theory, that tries to explain the beauty with mathematical language, aesthetic sense is achieved when the mind is able to detect a relative order, in a series of apparently non-regular and confused provocations.

There are several definitions for the aesthetics. Some, like Plato believed in the beauty of nature and the beauty of geometry, line and circle some like Plotin, contrary to Plato knew beauty something spiritual with the source of the soul. Aristotle gave objective sense to beauty. In ancient Greece (Hellenism school), personal creativity is important and architect becomes means of the expression of beauties of mathematics on the basis of harmony, symmetry and order. Peter Smith believes on three levels for aesthetic values; Fashion, design styles in different cultural periods, and aesthetic-cognitive core values that these three levels are changed over time according to the circumstances. According to Vitruvius (first century BC) and also Andrea Palladio (1508-1580), beauty along with efficiency and stability are three Designator factors in architecture and make a building laudable. In the words of Vitruvius, beauty can be achieved when the building has pleasant façade and the symmetry of its parts is calculated correctly. For Vitruvius v of symmetry is what we say today Proportionality. According to Kant (1724-1802), beauty is generally what makes our senses together in a concerted way. Accordingly, appreciation of the beauty of a form must be done without any intellectual work. According to Frank Lloyd Wright, beauty is a manifestation of the principled proportionality as line, form and color. Wright does not mention just the diversity of form and color in the aesthetic, but knows the legality of order, balance and unity in nature as the aesthetic factors. Adolf Loos also has the similar comments. For him, beauty is our highest evolution and the purpose that the human follows is that he seeks for aesthetic in form. [3]

Rudolf Arnheim expresses in his book The Dynamics of Architectural Form that "My main concern was that the visual expression is the necessary and really inevitable adjective of all architectural forms. [6] According to the definitions mentioned, the aesthetics can have principles such as order, harmony, symmetry, proportionality, balance, unity, etc. According to these principles, the aesthetics of the urban environments can be evaluated. The mentioned effective factors in aesthetics can manifest themselves in most of the visual forms.

\section{Form}

Form is the sensible aspect of a phenomenon. Form is the perceptible characteristic and identity of an object. Form is the manifestation of its constituent factors. We call the whole, substantiality and the appearance of a phenomenon formed with special materials, in order to express a theme, the meaning or content, as form. In addition to the role that form has in creating space, it has many main aspects. The first is the aesthetic of form that the appearance and characteristics of form are paid attention and so the subjects of scale, proportionality and shape (the result of difference of form from the base) are presented. The second is the relation of form and function and its effect on formation process of form and the third is the mean and content of form. Form is a mean that is mostly used for the expression of a symbolic concept and can express different meanings. Ability and capability to convert a subjective matter to form or in other words, to convert the idea to shape and form is one of the important parts in design process. [7]

All things and all places at all scales manifest their skeletal shapes similarly and according to the same rules. Phenomenon of the laws governing the skeletal forms refers to all cases, whether it is the urban landscape or a simple object that can be used daily. The empirical research conducted to understand the form aesthetic of the built environment, the emphasis of the studies on the relationship between complexity and simplicity, and enjoyable values of the environment. The relationship between being interesting and complexity, and complexity and being pleasant is shown in Figure 1. According to information theory and according to Arnheim (1977), if the levels are received above the regular complexity, patterns are evaluated more pleasant than the lack of regularity. [5]
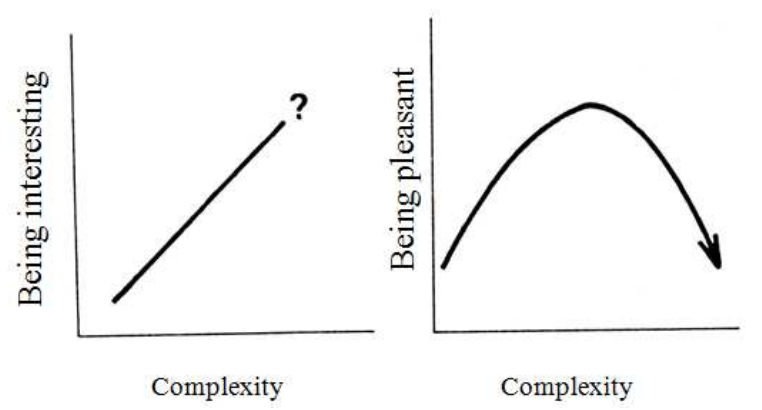

Figure 1. The relation between Complexity, Being interesting, Pleasure [5]. 
Forms as visual symbols to express a sense of meaning, are changeable symbols. The first step to understand the architecture work is form recognition. Form is the factor of creating space and removal of functional requirements. Form is objective by shape and other characteristics which are called "visual profile form". [8]

\subsection{Visual Characteristics of Form}

Constituent elements of form include point, line, surface, volume and color and architectural space is also affected by the relation between these elements and their combination. In order to combine and defining the relation between these elements, the builder elements such as geometry, symmetry, repetition, rhythm, axis and ... are presented. [7] For example, the application of line in the external form of a building can create special visual effects. In figure 2 , the left picture shows the façade of a building that is constituted using repeated and singular elements and owning static and without direction essence. In the right picture, applying lines and emphasizing on them connect the elements of the building to each other and gives them a horizontal essence, in addition, it also induces a sense of vitality and dynamism. [9]
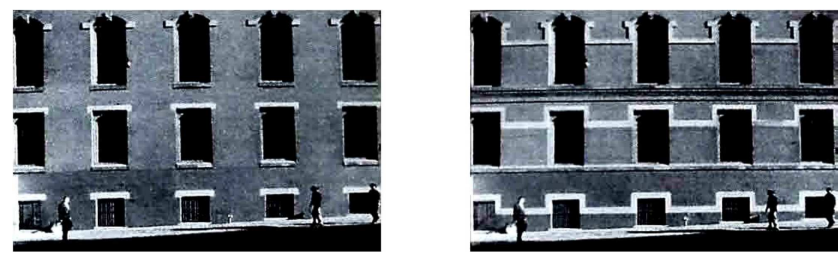

Figure 2. Applying lines and emphasizing on it in order to create sense of dynamism and visual mobility [9].

Each element, apart from the role and application that can have or its meaning, has visual characteristics and plays different manner and role in different situations and conditions [7] The visual characteristics of form include visual shape, size, color, texture, place, direction and balance.

Francis Ching knows shape as the most important identifier of form and says: "shape is called as the distance line of a visible surface or environment of a volume and the main means of detecting and identifying form of object." Length, width and height of form that are called dimensions, define the size and proportions of form. The other characteristic of form that in addition to its distinction from the surrounding environment that create different concepts and values in the viewer's mind is color. Strength of colors is hidden in their own characteristics. These characteristics are in the colors' darkness and clarity, coldness and warmth, saturation and level of surfaces area. Texture is presented in architecture by choosing different materials. Francis Ching introduces texture as the characteristic of the faces of form and knows it effective on the kind of the viewer's feeling and light reflection. The situation of a form in the environment relating to the existing forms surrounding is specified as the characteristic of that form. Ching says: "direction is considered as the position of form relating the ground level, surrounding points or relating a person looking at the form." Visual balance is a characteristic of form that expresses its stability or suspension. In fact, the balance is an equivalent perceived manner. This balance include stability and is applied in creating the sense of security and calmness. [8]

Each of volumes alone, like shapes, present different subjects. Heaviness, stability, purity, fluidity, fluency of form and ... are among the expressive characteristics of form. Domain of expressive characteristics and visual qualities increase in change and combination with each other and sometimes, the similarities to the known elements and forms add some meaning. The expressive abilities of a form is retrieved from seeming and physical characteristics of it. But part of these characteristics is defined dependent on its relation with other elements and the context that mostly, it is obvious and visible. But the meaning and content of each form is the characteristic hidden in a work and sometime, there is need to explore and understand. For example in figure 3, the form of the portal of Tehran University, the work of Kourosh Farzaami, present idea flight and symbolic pass with the presentation of two wings and vaulted form. [9]

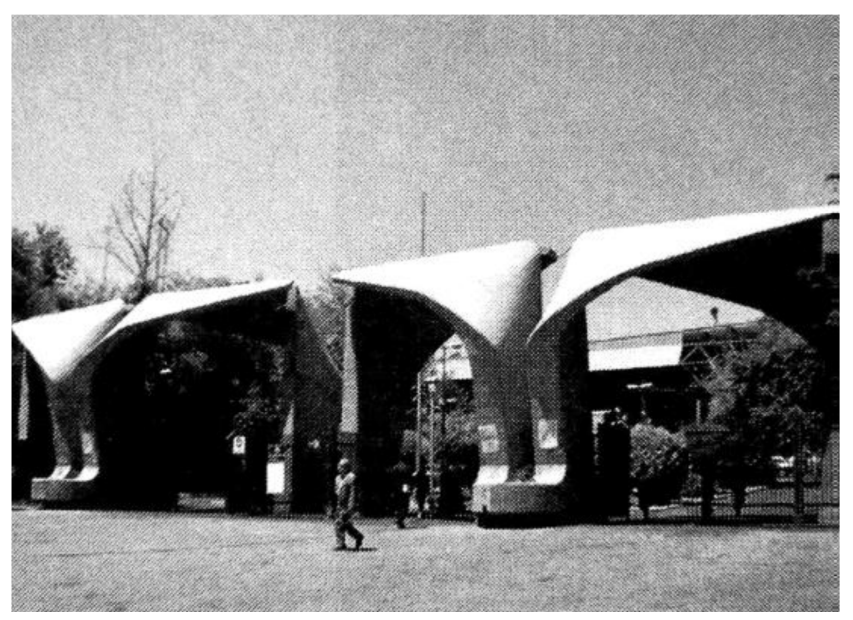

Figure 3. The portal of Tehran University, using a special form to express the concept of dynamic idea [7].

\subsection{The Characteristics Presenting Form and the Relation Between Forms}

There are characteristics presenting the form of volume, in all scales in the environment. Whether it is a building or a chair, in all cases, it is possible to analyze the characteristics of volumes such as heaviness or lightness, having a regular or irregular shape, existing an extra part in volume or overlapping of the parts. Each form, in small or big scale, in whole, has complexity in the consensus of forms hierarchy. This consensus can be presented in three shapes: the main form of element in the shape of a pure form, or the relation of many forms or organization of many forms on the base of a special regularity. The relation between forms and their effect on each other (figure 4) is shaped through several ways such as increasing forms on each other, subtracting a part from another part, overlapping of forms, dominance of an element on the other element, the contrast between elements, continuation, unity or division. [1] 


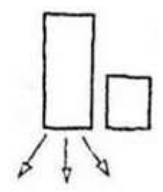

dominance

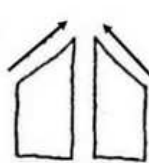

unity

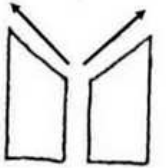

division

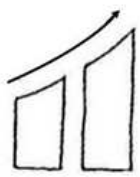

continuation
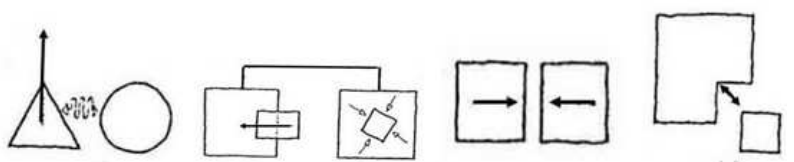

contrast iinvolvement overlapping increase

decreas
Figure 4. The relation between forms and their mutual influence on each other [1].

In the combination of the visual forms of environment, the most important issue that is firstly presented, is the element itself or its role. It means that which role the element has in the collection? Or what aims we have in using it? The second issue is the relation of element with the context and the third is the relation of elements with each other. Each form has other characteristics other than the seeming characteristics. These characteristics are the function and meaning of the form. This meaning, accompanying with fading the seeming characteristics, material and place, is emphasized and sometime, its discovery is very difficult. [7] To express any spiritual content, proportional and perceptible visual form should be created.

For example, the central forms express stability and silence, but the linear forms present dynamism and activity. [10] Using a special form in designing a space, it is possible to lead the viewer from less important spaces or the ones which want not to pay attention to, toward the pleasant space by creating hierarchy and giving direction to the space and viewer's vies in result. In figure 5, the simple house of Queen at the end of the visual axis is dominant in the heavy complex of buildings in both sides.

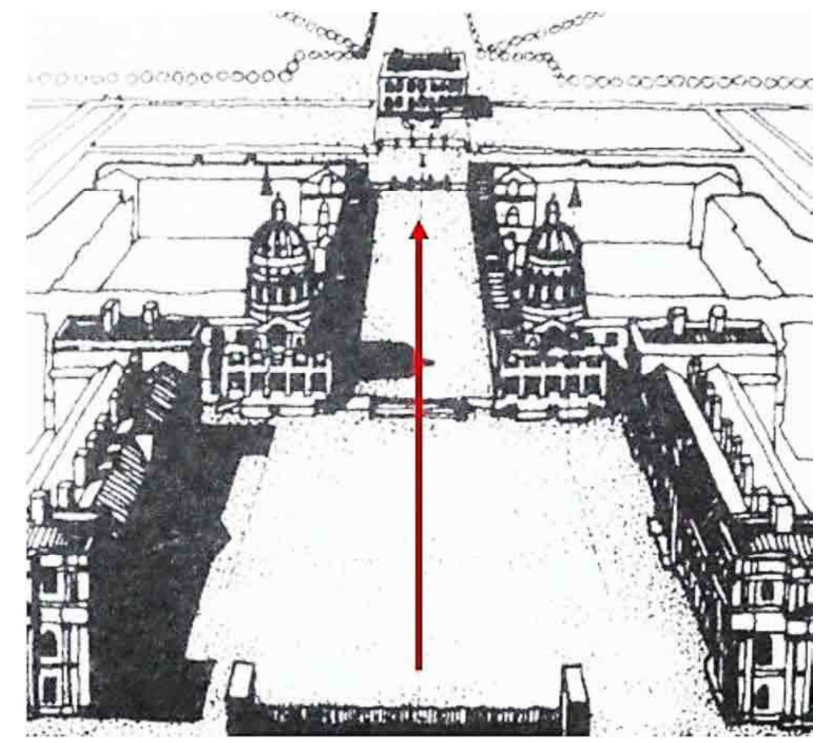

Figure 5. House of Queen, Greenwich, England, the effect of form and creating hierarchy in centralization and direction of the viewer [1].

\section{The Effect of Form in Aesthetics}

We reach to a meaning of a space by seeing (reading) the existing visual forms. According to this, the expression of contents using the visual forms is simpler than the spoken and written language. Since understanding the visual forms do not need decryption. For example, sometime, just seeing is enough to understand its function and gives us the necessary information to evaluate and understand it. [11]

The visual relation are not the mysterious phenomenon that are just perceptible by the professional but also they are simple characteristics. As example, the proportionate of the windows, position of the entrance, ornamental elements, style, material and skyline of the building in an urban environment are among the characteristics that are constituent of unity or lack of unity and integration of a street, neighborhood or region. (figure6). [12]

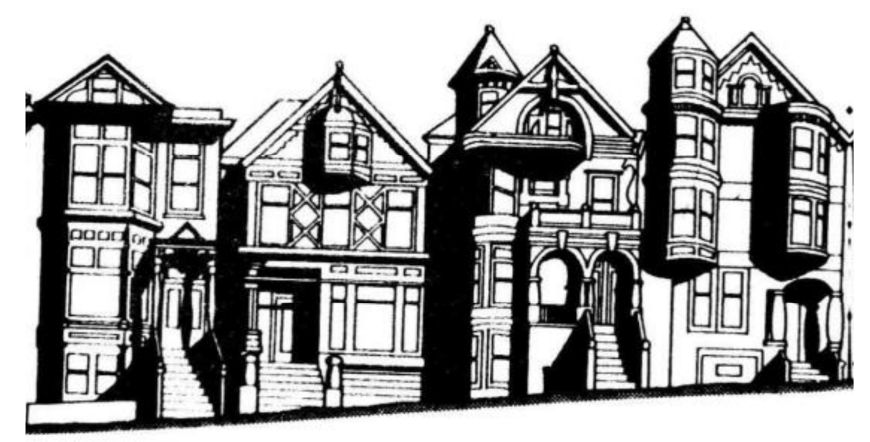

Figure 6. The visual relation and harmony [12].

Different visual forms can have different aesthetic effects on viewer. For example, the comparison of two building facades in figure 7 show that a large building with smooth façade, in the right picture, using color and texture that are among the constituent elements of form, is creatively converted to a narrower building that the content of visual mobility and dynamism is transmitted. [9]
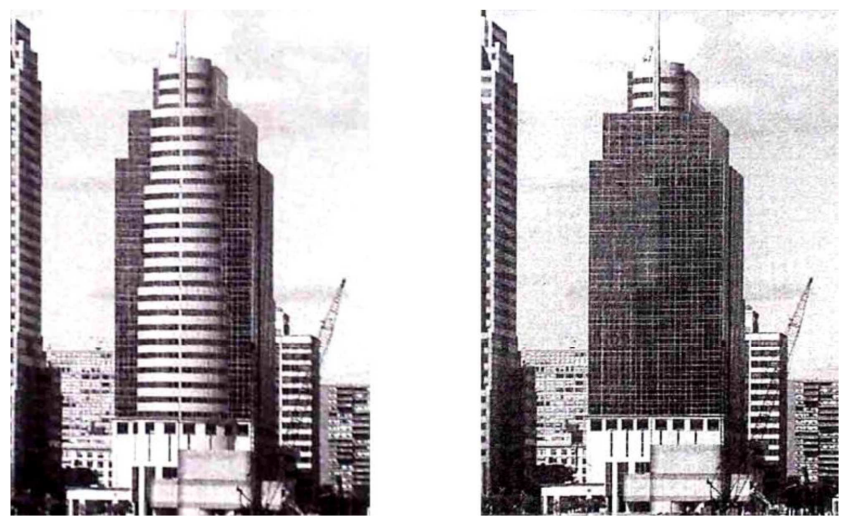

Figure 7. The effects of different visual forms on viewer using color and texture [9].

According to the mentioned aesthetic definition, principles such as proportionate, harmony, unity and ... are paid attention. Now, the question is that how and according to what forms locate next to each other? It is possible to find the answer of this question in the principles of aesthetics. In fact, 
the form must locate next to each other on the base of these principles.

Each urban environment have a combination of these elements and degree of freedom to create diversity in design. [12] So, the effect of form in aesthetics is very important. In the whole, whatever the relation and harmony between two elements is deeper, separating them from each other is more difficult. Certainly, between the elements having relation with each other, the searchable distance and cognition is desired so that the dialogue has meaning. [7] One of the ways of engagement, the relation and dialogue between two elements is insertion and subtraction of forms. [8]

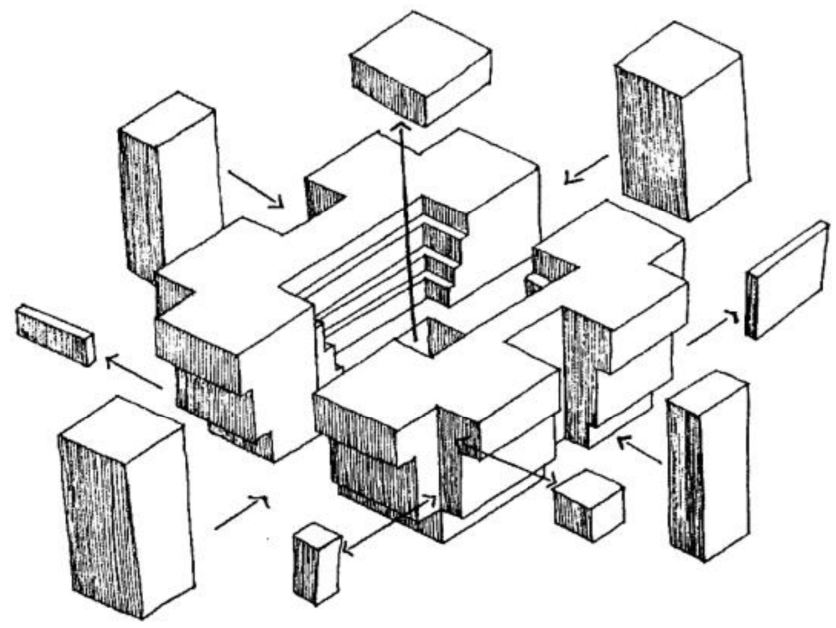

Figure 8. Insertion and subtraction [8].

The design of a form can be through the dimensional changes in length, width and height of the form. In the subtracted form, the totality has importance, but, in the inserted picture, this case is true and different inserted parts are important. [8] Symmetry that means harmony in Greek, suggest balance and proportions in aesthetic context. Locating two forms next to each other creates symmetry, while locating two different shape creates an apposed move. [1] For example, the problem of Frank Loyd Wright in designing Unitarian Church, is making relation between the square form of church and rectangular form of the side shape. (figure 9) wright makes involved two forms by continuing the side walls into the traces and solves the problem. Wright preserves the geometrical characteristics of primitive forms in the organization of elements)
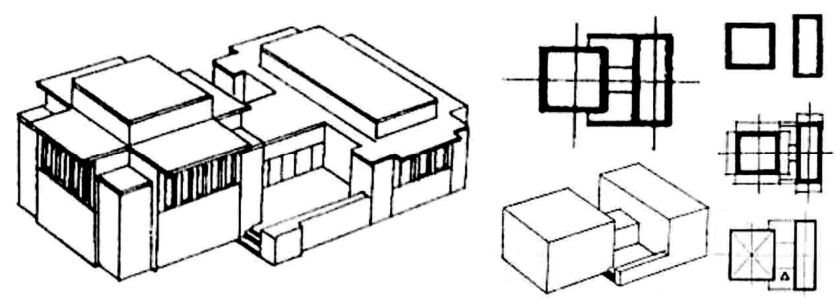

Figure 9. Unitarian Church, Oak Park, Illinois, Frank Loyd Wright 1906, How to combine two different forms [10].

How to locate the visual forms next to each other and how to combine them are among the issues that can be effective in reaching sustainability according to the aesthetic issue.

\section{Discussion and Conclusion}

City, in a wide scene, is constructing and renewing continuously. In the present urbanization, because of lack of coordinated criteria and principles in design, the city public space has become a heterogeneous structure and in aesthetics, and a chaotic freedom has dominated on construction. If the suitable, original and with identity about shape, geometry, skeletal texture of urban texture are not presented, the aesthetic essence of the culture of society is isolated and transformed.

In the definition of the urban sustainable spaces, paying attention to the aesthetic dimension of that environment, shows the design guidelines and solutions. Among the aesthetic solutions that can be named in order to reach the sustainability of an urban environment, are using proportions in the scale of visual elements of city environment, using diverse forms and suitable combination of them, creating visual rhythm and continuation, and their relation with each other and .... Factors such as sense place, dynamism, readability and ... that are cause of the sustainability of an urban environment, is possible by using some of these aesthetic principles and contents. If our goal in an urban environment is to move forward and toward a final point, the aesthetic guideline here introduces a form with the restrictions of the street sides and emphasize on leading by creating rhythm and continuation in the existing visual elements in environment. In result, each goal discovers an aesthetic guideline that this solution is manifested in the shape of a form.

\section{References}

[1] Tiss Evenson, T. Typology of Space, A Way to Aesthetical Design of Cities, Tehran Art University Pub. 2008.

[2] Golkar, K. Creation of Sustainable Place, Reflections on the Theory of Urban Design, Shahid Beheshti University Pub. 2011.

[3] Groter, Y. Aesthetics in Architecture, Shahid Beheshti University Pub. 2009.

[4] Nogrehkar, A. Introduction to Islamic identity in architecture and urbanism, Payame Sima Pub. 2008.

[5] Lang, J. Creation Architectural Theory, The Role of Behavioral Sciences in Environmental Design, Tehran University Pub. 2009.

[6] Arnheim, R. The Dynamics of Architectural Form, Tehran Pub. 2009.

[7] Arabi, J. Form Creation, Herfeye Honamand Pub. Tehran. 2009.

[8] Feyzi, M., Khakzand, M. Analyze of Ten Works from 50 Years of Iran Contemporary Architecture, Farhange Matin. 2010.

[9] Brolin, B. Architectural View, A Guideline to solve the Architectural Design Issues, Khak Pub. Esfehan Pub. 2003. 
[10] Ech Bicher, J. Le Corbusier, Form Analyze, Sobhane Nur Pub. 2007.

[11] A Denis, D. Basic Visual Literacy, Baztabe Andishe Pub. Tehran. 2007.
[12] Hedman, R. Yazouski, A. Fundamentals of Urban Design, Elm o Sanat University Pub. 2011. 